\title{
Impact of the Fiber Coating on the Temperature Response of Distributed Optical Fiber Sensors at Cryogenic Ranges
}

\author{
Xin $\mathrm{Lu}^{(\mathbb{B}}$, Marcelo A. Soto ${ }^{(0)}$, Member, IEEE, Member, OSA, and Luc Thévenaz ${ }^{\circledR}$, Fellow, IEEE, Fellow, OSA
}

\begin{abstract}
The thermomechanical behavior of a standard singlemode fiber with different coating materials is theoretically analyzed under different temperature conditions. Results show that the thermal expansion/shrinkage of the fiber coating introduces an extra strain on the optical fiber and can modify its thermal response. Distributed fiber sensors based on coherent Rayleigh and Brillouin scatterings are employed to characterize the impact of different coatings on the temperature sensitivity. The standard coating with dual-layer demonstrates a little influence on the thermal response at room temperature due to the softness of primary coating, but it increases the temperature sensitivity by some $50 \%$ at $\sim 220 \mathrm{~K}$ as the primary coating becomes stiffer at low temperature. Optical fibers with aluminum and Ormocer coatings are also experimentally tested. All the measured results agree well with the theoretical analysis.
\end{abstract}

Index Terms-Brillouin scattering, fiber optics, optical fiber sensors, Rayleigh scattering, thermomechanical process.

\section{INTRODUCTION}

A FTER 40 years of development, optical fiber sensors still attract researchers' interests due to their advantages over traditional sensors, such as small size, lightweight, low cost and resistance to electromagnetic interference [1]. Fiber sensors can be broadly classified in point and distributed sensors. Point (or discrete) sensors, such as Fabry-Perot interferometer and fiber Bragg grating (FBG), are able to measure the environmental variations at the very specific position where they are located. On the other hand, distributed fiber sensors (DFSs) utilize the whole fiber as a sensing medium and can perform continuous measurements of a physical variable (e.g., strain or temperature) over a long distance. So far, both types have been successfully commercialized in a wide range of application areas and are still under intensive development.

Manuscript received July 28, 2017; revised September 23, 2017; accepted September 25, 2017. Date of publication September 27, 2017; date of current version February 24, 2018. This work was performed within the framework of ITER-EPFL Task Agreement (Project Contract ITER/12/CT/4300000698). (Corresponding author: Xin Lu.)

$\mathrm{X}$. Lu was with the Institute of Electrical Engineering, Swiss Federal Institute of Technology of Lausanne, Lausanne 1015, Switzerland. He is now with the Institute for Astronomy, Swiss Federal Institute of Technology of Zürich, Zürich 8093, Switzerland (e-mail: luxin1026@gmail.com).

M. A. Soto and L. Thévenaz are with the Institute of Electrical Engineering, Swiss Federal Institute of Technology of Lausanne, Lausanne 1015, Switzerland (e-mail: marcelo.soto@epfl.ch; luc.thevenaz@epfl.ch).

Color versions of one or more of the figures in this paper are available online at http://ieeexplore.ieee.org.

Digital Object Identifier 10.1109/JLT.2017.2757843
Distributed optical fiber sensors exploit essentially the natural scattering phenomena occurring inside an optical fiber (e.g., Raman, Brillouin, and Rayleigh scattering processes). For Raman sensing, the temperature profile along the fiber is retrieved from the optical power ratio between the anti-Stokes and Stokes (or Rayleigh) waves [2]. In the case of a Brillouin distributed sensor, the local Brillouin frequency shift (BFS) is measured to retrieve the environmental information [3]. DFSs based on coherent Rayleigh scattering respond in a manner similar to a FBG sensor, but in a distributed way: its reflection response shifts spectrally due to variations of environmental conditions [4]. All three kinds of DFSs have achieved great success working around room temperature $(\sim 300 \mathrm{~K})$, but they typically demonstrate a reduced sensing performance in cryogenic temperature environments. Spontaneous Raman scattering is a thermallyactivated process and therefore the corresponding anti-Stokes component vanishes at low temperature [5]. On the other hand, the temperature sensitivity of Brillouin sensors nullifies around $80 \mathrm{~K}$ [6]. According to the FBG sensing theory [7], the thermal response of a coherent Rayleigh sensor is dominated by thermooptic effect, which becomes weak under cryogenic condition, so its temperature sensitivity decreases as the temperature turns low.

Whilst Raman sensors are only temperature-dependent, a relevant feature of Brillouin and Rayleigh distributed sensors is that their response depends on both temperature and strain, resulting in a cross-sensitivity that makes it impossible to discriminate one variable from another by a simple measurement procedure. Interestingly, this cross-sensitivity turns out to be beneficial to improve the temperature sensitivity of Rayleigh and Brillouin sensors, because the thermal expansion or shrinkage of the fiber coating leads to a thermally-induced strain in the optical fiber, hence enhancing the influence of temperature changes on the fiber [8]. Actually, a variety of metals and polymers with large positive coefficient of thermal expansion (CTE) have been used as fiber coatings to enhance the thermal response of different fiber sensors, particularly at low temperature [7]-[12]. Note that the strain-independent response of spontaneous Raman scattering in silica fibers makes the thermally-induced strain to have no impact on the performance of Raman sensing.

Despite the extensive research on applications of special coatings for fiber sensing, the single mode fiber (SMF) with a standard fiber coating is still the most widely used in distributed fiber sensing. The standard coating usually consists of two layers: a 


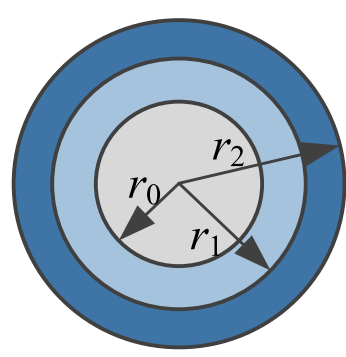

(a)

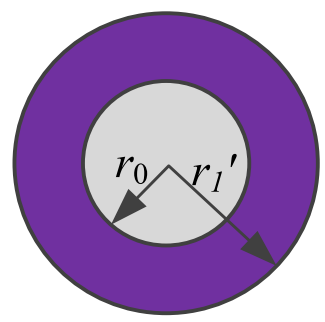

(b)
Fig. 1. Cross-section of single mode fiber with (a) standard coating; (b) special coating. Gray: fiber glass, light blue: primary coating, dark blue: secondary coating, purple: special coating.

strong outer layer (secondary coating) protects the fiber and a soft inner layer (primary coating) adheres the outer layer and the optical fiber together [13]. Actually, the CTE of this coating is also high, but it demonstrates almost no impact on the thermal response of the fiber, because the soft inner layer hinders the thermally-induced strain transferred from the coating to the silica fiber. However, as the inner layer becomes stiffer at low temperature, the impact of the thermal expansion of the coatings can no longer be neglected [13], and a higher temperature sensitivity can be expected.

In this paper, the impact of the thermally-induced strain on the temperature response of Brillouin and Rayleigh distributed sensors is investigated theoretically and experimentally based on the thermomechanical behavior of SMFs with different coatings. The contribution of this thermomechanical behavior to the temperature sensitivity of DFSs is investigated experimentally in a wide temperature range, from $\sim 220 \mathrm{~K}$ up to room temperature $(\sim 300 \mathrm{~K})$. The thermal response of fibers with standard, aluminum or Ormocer coating is experimentally characterized by Brillouin and coherent Rayleigh sensing techniques. A large temperature sensitivity enhancement at $\sim 220 \mathrm{~K}$ is experimentally confirmed for the fiber with standard coating, whilst all experimental results agree well with the theoretical analysis for different coatings under distinct thermal conditions.

\section{THEORETICAL ANALYSIS}

\section{A. Thermomechanical Model}

Standard fibers are usually coated by two layers of polymers, with a cross-section as shown in Fig. 1(a). The dark blue section represents the secondary coating, which is strong enough to protect the fiber from mechanical damage [13]. The primary coating, inner layer, is however made of soft visco-elastic materials in order to reduce the attenuation caused by microbending. The Young's modulus of the inner layer is very low at room temperature but increases by three-order of magnitude at the glass transition point around $220 \mathrm{~K}$ [13]. Therefore, the thermal expansion of the coatings at room temperature shows a very limited impact on the opto-thermal response of the fiber in spite of the large CTE of the coating. However, the primary coating starts to exhibit a glass behavior at low temperatures, resulting in a non-negligible thermally-induced strain over the silica fiber, directly impacting the temperature sensitivity of the fiber sensors. On the other hand, special coatings usually adhere directly to the optical fiber, as shown in Fig. 1(b). Therefore, the thermal expansion of the coating transfers directly to the fiber, modifying the thermal response of the sensor. Hence special coatings usually have a clear impact on the thermal response even at room temperature.

Based on the reported thermomechanical model [10], [14], the thermally-induced strain in the optical fiber with standard coating can be calculated. The optical fiber, primary and secondary coatings demonstrate different responses to the same temperature change $\Delta T$ because of their different physical properties. Thus the thermally-induced stresses in radial, tangential, axial dimensions $\left[\sigma_{r}^{i}, \sigma_{\theta}^{i}, \sigma_{z}^{i}\right]$ of each layer can be described by the Lamé solutions [14]:

$$
\left[\begin{array}{c}
\sigma_{r}^{i} \\
\sigma_{\theta}^{i} \\
\sigma_{z}^{i}
\end{array}\right]=\left[\begin{array}{ccc}
\xi_{i}+2 \mu_{i} & \xi_{i} & \xi_{i} \\
\xi_{i} & \xi_{i}+2 \mu_{i} & \xi_{i} \\
\xi_{i} & \xi_{i} & \xi_{i}+2 \mu_{i}
\end{array}\right]\left[\begin{array}{c}
\varepsilon_{r}^{i}-\alpha_{i} \Delta T \\
\varepsilon_{\theta}^{i}-\alpha_{i} \Delta T \\
\varepsilon_{z}^{i}-\alpha_{i} \Delta T
\end{array}\right]
$$

where $i=0,1,2$ represents the different layers, $\alpha_{i}$ is the linear $\mathrm{CTE}, \xi_{i}$ and $\mu_{i}$ are the Lamé parameters, expressed as:

$$
\begin{aligned}
\xi_{i} & =\eta_{i} E_{i} /\left[\left(1+\eta_{i}\right)\left(1-2 \eta_{i}\right)\right] \\
\mu_{i} & =E_{i} /\left[2\left(1+\eta_{i}\right)\right]
\end{aligned}
$$

respectively, with the corresponding Poisson's ratio $\eta_{i}$ and Young's modulus $E_{i}$. The factors $\varepsilon_{r}^{i}$, $\varepsilon_{\theta}^{i}$, and $\varepsilon_{z}^{i}$ in Eq. (1) represent the corresponding strains in radial, tangential, axial dimensions, written as:

$$
\varepsilon_{r}^{i}=U_{i}+V_{i} / r^{2}, \varepsilon_{\theta}^{i}=U_{i}-V_{i} / r^{2}, \varepsilon_{z}^{i}=W_{i}
$$

where $U_{i}, V_{i}$ and $W_{i}$ are constants that can be determined by the boundary conditions. The continuity between different layers leads to:

$$
\begin{aligned}
& \sigma_{r}^{i}\left(r_{i}\right)=\sigma_{r}^{i+1}\left(r_{i}\right) \quad i=0,1 \\
& \mu_{r}^{i}\left(r_{i}\right)=\mu_{r}^{i+1}\left(r_{i}\right) \quad i=0,1
\end{aligned}
$$

where $r_{i}$ is the radius of the $i$ th layer. Assuming that the fiber experiences no external forces, then

$$
\sigma_{r}^{2}\left(r_{2}\right)=0, \quad \sum_{i=0}^{2} \sigma_{z}^{i} \cdot A_{i}=0
$$

where $A_{i}$ represents the cross-section area of the $i$ th layer.

Since the fiber can be considered as a long and thin cylinder, a plain strain approximation can be applied, so that [10], [14]

$$
\varepsilon_{z}^{0}=\varepsilon_{z}^{1}=\varepsilon_{z}^{2}=\varepsilon_{z} .
$$

Due to the axial symmetry of the optical fiber, the thermallyinduced strain in radial direction $\varepsilon_{r}^{0}$ and in tangential dimension $\varepsilon_{\theta}^{0}$ must keep finite at the fiber center $r=0$, resulting in $V_{0}=0$ if the thermal load is also assumed axisymmetric.

This model can be easily adapted to analyze the fiber with special coating by neglecting the secondary layer and changing the boundary conditions accordingly. 
TABLE I

Relevant Physical Parameters of a Standard Single Mode Fiber AND ITS DUAL-LAYER ACRYLATE COATING

\begin{tabular}{cc|cc|cc}
\hline$r_{0}$ & $62.5 \mu \mathrm{m}$ & $\eta_{0}$ & $0.17[10]$ & $E_{0}$ & $72 \mathrm{GPa}[10]$ \\
$r_{1}$ & $95 \mu \mathrm{m}$ & $\eta_{1}$ & $0.4995[10]$ & $E_{2}$ & $1 \mathrm{GPa}[18]$ \\
$r_{2}$ & $125 \mu \mathrm{m}$ & $\eta_{2}$ & $0.452[17]$ & $a_{2}$ & $7.93 \times 10^{-5} \mathrm{~K}^{-1}[15]$ \\
\hline
\end{tabular}

Based on the boundary conditions listed in Eqs. (3)-(6), the thermally-induced strain in the optical fiber can be numerically calculated with the corresponding fiber parameters. As mentioned above, the Young's modulus of the primary coating $E_{1}$ increases significantly at the glass transition temperature, from $1 \mathrm{MPa}$ at room temperature to $\sim 2.6 \mathrm{GPa}$ at $\sim 220 \mathrm{~K}$ [13]. The CTE of the primary coating $\alpha_{1}$ is measured to be $2.11 \times 10^{-4} \mathrm{~K}^{-1}$ at room temperature and becomes $1.18 \times 10^{-4} \mathrm{~K}^{-1}$ in the temperature range from $223 \mathrm{~K}$ to $173 \mathrm{~K}$ [15]. In addition, according to the experiment on fused silica SRM 739 [16], the CTE of the optical fiber $\alpha_{0}$ is $0.45 \times 10^{-6} \mathrm{~K}^{-1}$ at room temperature and decreases down to $0.21 \times 10^{-6} \mathrm{~K}^{-1}$ at $\sim 220 \mathrm{~K}$. Other relevant parameters of standard SMFs, assumed temperature-independent, are listed in Table I.

In this paper, only the strains in axial and radial directions are considered because they have been proven to have a dominant influence on the thermally induced optical phase change in optical fibers [14]. At room temperature, the thermally-induced strains $\Delta \varepsilon_{z}$ and $\Delta \varepsilon_{r}^{0}$ are calculated to be $1.78 \mu \varepsilon$ and -0.09 $\mu \varepsilon$ per $1 \mathrm{~K}$ increase, respectively. Such small strains are due to the soft primary coating which prevents the fiber from the thermal expansion of the coating. However, large strains can be expected when the primary coating shows a glass-like response. A numerical analysis shows that a $1 \mathrm{~K}$ increase at $\sim 220 \mathrm{~K}$ introduces strains of $7.41 \mu \varepsilon$ and $-0.46 \mu \varepsilon$ along axial and radial directions, respectively. The large strain is supposed to have an obvious impact on the thermal response of fiber sensors based on Brillouin and coherent Rayleigh scatterings, as it will be demonstrated hereafter.

It has to be pointed out that the temperature change may also introduce micro-bending to the fiber because of the thermal expansion mismatch between fiber glass and coating material, which is neglected in this paper. This is valid for the standard double-layer coating system at room temperature because the soft primary coating highly suppresses the micro-bending [13], [19]. However, the primary coating becomes stiffer when the temperature is below the glass transition point $(\sim 220 \mathrm{~K})$, so the micro-bending loss will increase [19] and may lead to lower trace SNR, ultimately undermining the performance of the sensing system.

\section{B. Temperature Sensitivity Enhancement for SMF With Standard Coating}

For Brillouin distributed fiber sensing, the local BFS $f_{B}$ is measured along the fiber as a function of distance. This BFS depends on the acoustic velocity of silica, which turns out to be dependent on temperature and strain. Therefore, temperature and strain variations cause a BFS change $\Delta f_{B}$, expressed as:

$$
\Delta f_{B}=S_{T}^{B} \cdot \Delta T+S_{\varepsilon, z}^{B} \cdot \Delta \varepsilon_{z}+S_{\varepsilon, r}^{B} \cdot \Delta \varepsilon_{r}^{0}
$$

where $S_{T}^{B}, S_{\varepsilon, r}^{B}$ and $S_{\varepsilon, z}^{B}$ are the temperature, radial and axial strains sensitivities, respectively.

The temperature sensitivity $S_{T}^{B}$ is a constant of $\sim 1 \mathrm{MHz} / \mathrm{K}$ from room temperature down to $150 \mathrm{~K}$ at a working wavelength around $1550 \mathrm{~nm}$ [20]. The radial and axial strain sensitivities of a Brillouin sensor are measured to be $0.05 \mathrm{MHz} / \mu \varepsilon$ and $0.029 \mathrm{MHz} / \mu \varepsilon$, respectively, at room temperature for a standard SMF [21]. In addition, no large variations of $S_{\varepsilon, z}^{B}$ are observed in the temperature range from $200 \mathrm{~K}$ to $300 \mathrm{~K}$ [22] and the radial sensitivity $S_{\varepsilon, r}^{B}$ is assumed to have the same behavior.

Equation (7) shows that the thermally-induced strain contributes to the BFS change, so that the actual temperature sensitivity can be rewritten as $S_{T}^{B^{\prime}}=\Delta f_{B} / \Delta T$. Considering $S_{T}^{B}$, $S_{\varepsilon, r}^{B}, S_{\varepsilon, z}^{B}$ and the extra thermally-induced strains analyzed in the previous section, the conventional temperature sensitivity of $1 \mathrm{MHz} / \mathrm{K}$ in a standard SMF is increased by $0.09 \mathrm{MHz} / \mathrm{K}$ and $0.36 \mathrm{MHz} / \mathrm{K}$ at $300 \mathrm{~K}$ and $220 \mathrm{~K}$, respectively. The temperature sensitivity increase is quite small at room temperature as expected, owing to the low Young's modulus of the primary layer. But since the primary coating becomes stiffer at $220 \mathrm{~K}$, it enables the strain transfer from the coatings to the fiber, resulting in a significant temperature sensitivity enhancement.

To realize distributed sensing exploiting coherent Rayleigh scattering, the Rayleigh reflection spectrum is acquired along the fiber by recording the local signal intensity within a certain optical frequency range. The stochastic density fluctuations along the fiber lead to a random longitudinal profile of refractive index, which partially reflects the incident light. Hence, the fiber used in the coherent Rayleigh sensor is usually modeled as a long, weak FBG with random amplitude and pitch [23], [24]. The light backscattered within the pulse width interferes randomly, resulting in irregular temporal and spectral shapes. Environmental variations causing changes of the fiber refractive index, such as temperature and strain, induce a spectral shift $\Delta f_{R}$. The frequency shift is usually determined by crosscorrelating the spectra measured in two different situations (a reference, and a current measurement condition) [25]. Coherent Rayleigh sensors actually work exactly following the same principle as a FBG, and therefore its working principle can be fully described by the well-known and well-established grating theory. For a FBG, the Bragg wavelength change $\Delta \lambda_{B}$ induced by temperature and strain variations is expressed as [26]:

$$
\begin{aligned}
\Delta \lambda_{B} & =\lambda_{B}\left(\begin{array}{l}
\left\{1-n^{2} / 2 \cdot\left[P_{12}-\eta_{0}\left(P_{11}+P_{12}\right)\right]\right\} \varepsilon_{z} \\
+\left(\alpha_{0}+\frac{1}{n} \frac{d n}{d T}\right) \Delta T
\end{array}\right) \\
& =\lambda_{B}\left(S_{\varepsilon}^{R} \varepsilon_{z}+S_{T}^{R} \Delta T\right)
\end{aligned}
$$

where $\lambda_{B}$ represents the Bragg wavelength, $n$ is the refractive index of the fiber, $P_{11}$ and $P_{12}$ are Pockel's coefficients of the stress-optic tensor, the factor $\left\{1-n / 2\left[P_{12}-\eta_{0}\left(P_{11}+P_{12}\right)\right]\right\}$ is taken as $0.22, \mathrm{~d} n / \mathrm{d} T$ represents the thermo-optic coefficient of silica, and $S_{T}^{R}$ and $S_{\varepsilon}^{R}$ are the corresponding temperature and strain sensitivities, respectively. The refractive index of the fiber 
can be calculated using the Sellmeier expansion under different temperature conditions, so that the thermo-optic coefficient is obtained accordingly [27]. It has to be pointed out that Eq. (8) considers strains not only in the axial direction, but also along the radial direction through the Poisson's ratio.

The frequency shift of the reflection spectrum in coherent Rayleigh sensing can be easily obtained by converting the wavelength change $\Delta \lambda_{B}$ into frequency. As a result, the temperature sensitivity is calculated to be $-1.22 \mathrm{GHz} / \mathrm{K}$ at room temperature and $-0.98 \mathrm{GHz} / \mathrm{K}$ at $227 \mathrm{~K}$ when using a light source at $1534 \mathrm{~nm}$. On the other hand, the strain sensitivity of a FBG is measured as $-150 \mathrm{MHz} / \mu \varepsilon$ and has been proved to be temperature independent [28]. Considering the thermally-induced strains obtained above, the temperature sensitivity for a coherent Rayleigh sensor increases up to $-1.48 \mathrm{GHz} / \mathrm{K}$ and $-2.02 \mathrm{GHz} / \mathrm{K}$ at $300 \mathrm{~K}$ and $227 \mathrm{~K}$, respectively. Actually, a large sensitivity at low temperature in coherent Rayleigh sensing has been already reported in the literature [29]; however, this behavior has never been fully explained from the theoretical point of view.

\section{EXPERIMENTAL RESULTS AND DISCUSSION}

\section{A. Experiments on SMF With Standard Coating}

To validate the temperature sensitivity enhancement for distributed sensing, a Brillouin optical time domain analyzer (BOTDA) [3] and a coherent Rayleigh optical time domain reflectometer (COTDR) [4], [29] are set up to test a 14-m long SMF with standard coating. A laser working around $1534 \mathrm{~nm}$ is employed as a light source in the two cases. Optical pulses with high extinction ratio are generated with a width of $17 \mathrm{~ns}$, corresponding to $1.7 \mathrm{~m}$ spatial resolution. The sensing fiber has been loosely coiled inside a copper box so that the longitudinal temperature gradients along the sensing fiber are highly suppressed. For room temperature measurements, the box has been placed in a thermal bath with a tunability of $0.1 \mathrm{~K}$, so that a uniform temperature distribution along the entire sensing fiber is obtained thanks to the large heat capacity of water. In addition, the thermal exchange between water and ambient air is highly reduced, providing stable temperature conditions for the measurements. On the other hand, liquid nitrogen is used for low temperature sensing, whilst large temperature changes are realized by adjusting the copper box elevation above the liquid nitrogen level.

The local BFS is determined by parabolic fitting of the Brillouin gain spectrum [30]. The BFS averaged along the sensing fiber is plotted as a function of temperature in Fig. 2, where two distinct regions are clearly observed. The corresponding temperature sensitivity is $1.1 \mathrm{MHz} / \mathrm{K}$ from $260 \mathrm{~K}$ to $300 \mathrm{~K}$, very close to the theoretical result $\sim 1.05 \mathrm{MHz} / \mathrm{K}$. On the other hand, a steeper slope is observed around $220 \mathrm{~K}$, representing a higher sensitivity $(1.5 \mathrm{MHz} / \mathrm{K})$. The theoretical value expected from the analysis in Section II $(\sim 1.36 \mathrm{MHz} / \mathrm{K})$ is about $10 \%$ lower than the measurement result, which might be explained by discrepancies between coating parameters (e.g., composition and thickness) used in the theoretical analysis and those of the real fiber employed in the experiment. Experimental result confirms

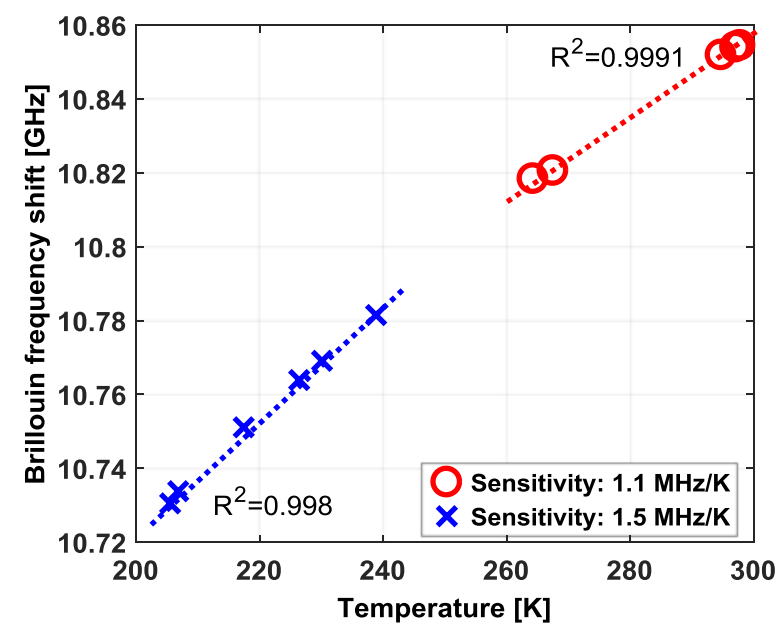

Fig. 2. Brillouin frequency shift of a single mode fiber with a standard coating at different temperatures. A temperature sensitivity enhancement is observed around $\sim 220 \mathrm{~K}$ due to the thermal expansion of the fiber coating.

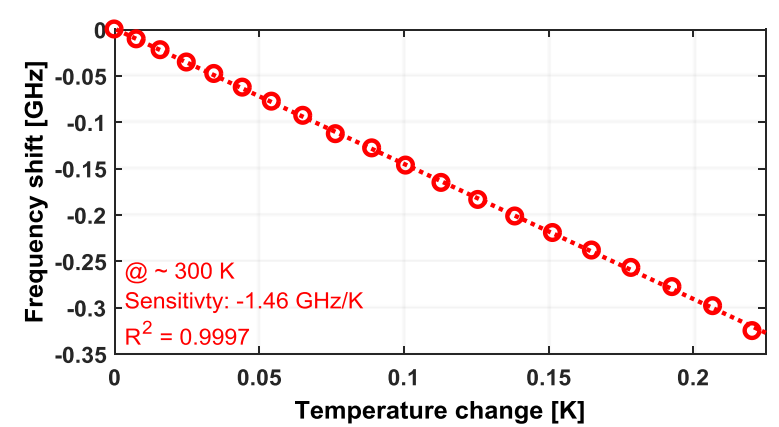

(a)

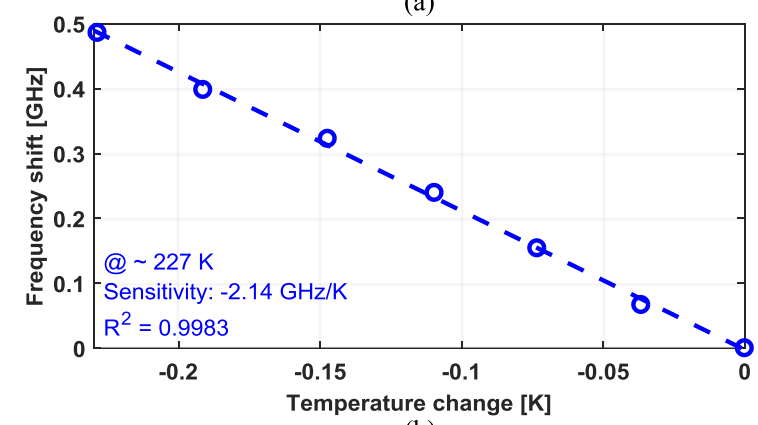

(b)

Fig. 3. COTDR response of a single mode fiber with standard dual-layer coating at (a) room temperature and (b) low temperature (around $227 \mathrm{~K}$ ).

the temperature sensitivity enhancement in Brillouin distributed sensing at low temperatures. Note that this phenomenon has never been reported before, presumably because the BFS behavior versus temperature is commonly linearly fitted within the entire range from $150 \mathrm{~K}$ to $300 \mathrm{~K}$, so the observation of the temperature sensitivity enhancement around $\sim 220 \mathrm{~K}$ has been simply skipped. For example, all the data in Fig. 2 can still be fitted with a good linearity $\left(\mathrm{R}^{2}=0.996\right)$.

The experimental results obtained by COTDR under different temperature conditions are presented in Fig. 3. At room temperature, the temperature sensitivity reaches $-1.46 \mathrm{GHz} / \mathrm{K}$ as shown 


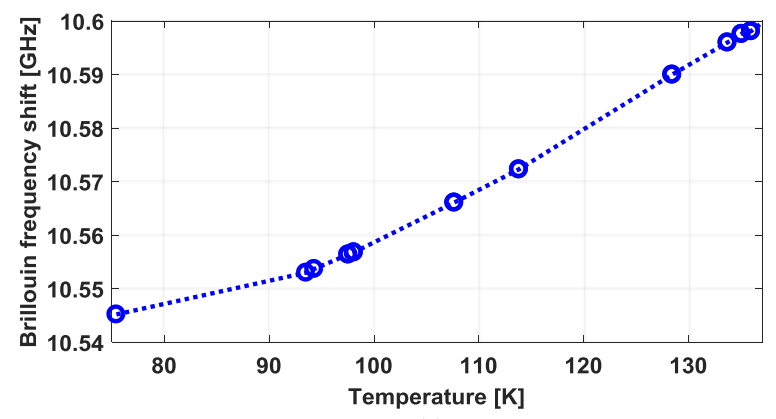

(a)

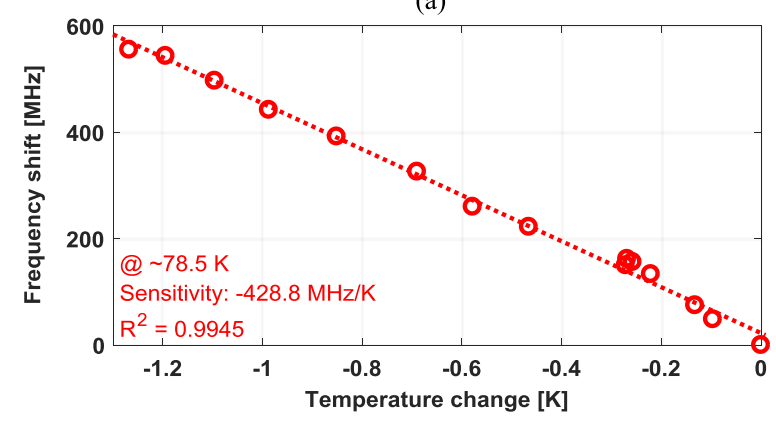

(b)

Fig. 4. Thermal responses of a single mode fiber with standard dual-layer coating measured by (a) Brillouin sensor and (b) coherent Rayleigh sensor below $220 \mathrm{~K}$.

in Fig. 3(a), agreeing very well with the theoretical analysis of Section II. As expected an even higher sensitivity is observed around $\sim 227 \mathrm{~K}$ in Fig. 3(b) due to the thermally-induced coating strain, despite the decrease of the thermo-optic coefficient as the temperature reduces. In this case, the theoretical analysis underestimates the sensitivity by $\sim 13 \%$.

Figs. 2 and 3 demonstrate that both measured frequency shifts $\Delta f_{\mathrm{B}}$ and $\Delta f_{\mathrm{R}}$ have good linear relations with temperature under different thermal environments. Experimental results match well with the theoretical predictions at room temperature for both sensing methods, but they turns to be $\sim 10 \%$ higher at $\sim 220 \mathrm{~K}$ compared to the theoretical analysis in Section II, which might also be explained by the inaccurate information about the actual coating parameters.

It has to be pointed out that the thermally-induced strain in the coating demonstrates its largest impact on the thermal response at $\sim 220 \mathrm{~K}$ because the Young's modulus of the primary coating stops increasing as the temperature reduces. Therefore, the temperature sensitivities of the two distributed fiber sensors become weak again below $220 \mathrm{~K}$, as shown in Fig. 4, because of the smaller acoustic velocity change with temperature for the Brillouin sensor [20] and the lower thermo-optic coefficient of silica for the coherent Rayleigh sensor [29].

\section{B. Experiments on SMF With Special Coatings}

The impact of special coatings (metal and polymer) on the thermal response of the DFS is also investigated at room temperature in order to validate the thermomechanical model. Al and Ormocer coatings, with parameters shown in Table II, are chosen to represent metal and polymer, respectively. It has to be
TABLE II

ReleVant Physical Parameters of ORMocer AND Al COATINGS AT ROOM TEMPERATURE

\begin{tabular}{l|l|l}
\hline & Ormocer $^{\circledR}$ & $\mathrm{Al}$ \\
$r_{1}$, & $97.5 \mu \mathrm{m}$ & $87.5 \mu \mathrm{m}$ \\
$\eta_{1}$, & $0.49[31]$ & $0.33[32]$ \\
$E_{1}$, & $1.5 \mathrm{GPa}[31]$ & $70 \mathrm{GPa}[32]$ \\
$\alpha_{1}$, & $1.15 \times 10^{-4} \mathrm{~K}^{-1}$ & $2.3 \times 10^{-5} \mathrm{~K}^{-1}[32]$ \\
\hline
\end{tabular}

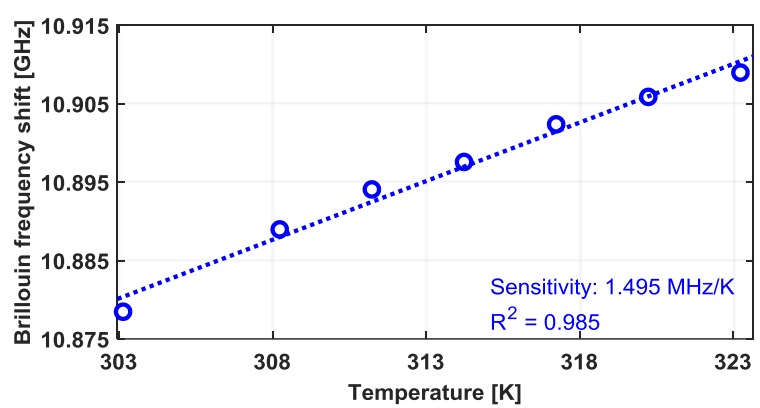

(a)

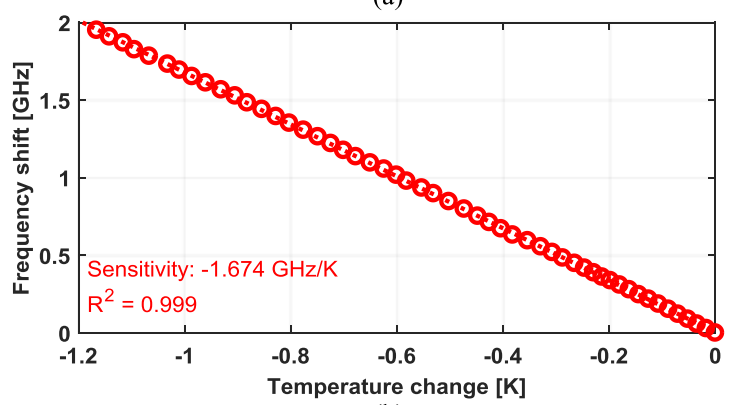

(b)

Fig. 5. Thermal response of (a) the Al-coated fiber measured by Brillouin sensing and (b) Ormocer coated fiber measured by coherent Rayleigh sensing at room temperature.

noted that the CTE of Ormocer is usually in the range from 1 to $1.3 \times 10^{-4} \mathrm{~K}^{-1}$, so that a mean value is used in this case. Thus, the thermally-induced strain can be calculated based on the thermomechanical model. Consequently, temperature sensitivity of the Rayleigh sensor is enhanced up to $-1.759 \mathrm{GHz} / \mathrm{K}$ by the Ormocer coating and the Al coating can improve the sensitivity of Brillouin sensing by $0.538 \mathrm{MHz} / \mathrm{K}$.

The Al-coated fiber sample turns out to be too short $(1.3 \mathrm{~m})$ to carry out a meaningful COTDR measurement, so that the thermal response of this fiber is only characterized by Brillouin sensing. A double-pulse BOTDA scheme is implemented to achieve sub-meter resolution [33]. Short pump pulses (2 ns) are used to reach $20 \mathrm{~cm}$ spatial resolution. Note that the Al coating thickness varies within $\pm 9 \mu \mathrm{m}$ along the sample, so that the thermally-induced strain is not fully uniform along the entire fiber. Fig. 5 shows the average BFS value over the fiber, at ambient temperature. The obtained sensitivity is $1.495 \mathrm{MHz} / \mathrm{K}$, matching very well the theoretical prediction.

To complete the picture, the thermal response of the Ormocer coated fiber is characterized using a COTDR system. Fig. 5(b) demonstrates that the frequency shift of the cross-correlated peak obtained in a coherent Rayleigh sensor has a good linear 
relationship with the temperature change, showing a sensitivity very close to the theoretically expected result (only $\sim 5 \%$ difference). A similarly enhanced response is expected for a Brillouin measurement.

\section{CONCLUSION}

In this paper, the impact of different fiber coatings on the thermal response of DFSs has been theoretically analyzed making use of a thermomechanical model and experimentally validated by coherent Rayleigh and Brillouin sensing techniques at room and low temperatures. Experimental results demonstrate that the thermal expansion/shrinkage of the coating with large positive CTE results in a thermally-induced strain that transfers to the fiber and enhances the temperature sensitivity. In particular, in a standard dual-layer fiber coating, the temperature sensitivity increases at around $\sim 220 \mathrm{~K}$ when the primary coating increases its stiffness through a glass transition, while its influence is almost negligible at ambient temperature conditions due to the small Young's modulus of the primary coating. Consequently, special attention must be paid when using Rayleigh and Brillouin distributed optical fiber sensors at low temperatures, where a simple extrapolation from ambient response can lead to an erroneous predicted response. On the other hand, the SMFs with metallic coatings demonstrate enhanced thermal response even at room temperature. All the experimental results match the theoretical analysis reasonably, considering the large uncertainty in the real coating parameters.

Theoretical and experimental results demonstrate that the temperature sensitivity of DFS based on coherent Rayleigh and Brillouin scatterings can be adjusted using different coatings, so that an enhanced temperature sensitivity can be realized at cryogenic environment. In addition, the two distributed sensing techniques have the potential to measure the longitudinal nonuniformity of the fiber coating by measuring the local thermal response of the fiber.

\section{ACKNOWLEDGMENT}

The authors would like to thank Dr. H. Limberger at EPFL, for clarifying helpful discussions on fiber coatings, Dr. K. Schuster at IPHT Jena, for providing the Ormocer-coated fiber sample, and AMS Technologies and Fiberguide for providing the Alcoated fiber sample used in this experiment.

\section{REFERENCES}

[1] F. Yu and S. Yin, "Overview of fiber optic sensors," in Fiber Optic Sensors, 1st ed. New York, NY, USA: Marcel Dekker, 2002, pp. 1-38.

[2] G. Bolognini and A. Hartog, "Raman-based fibre sensors: Trends and applications," Opt. Fiber Technol., vol. 19, no. 6, pp. 678-688, Sep. 2013.

[3] A. Motil, A. Bergman, and M. Tur, "State of the art of Brillouin fiber-optic distributed sensing," Opt. Laser Technol., vol. 78, pp. 81-103, Apr. 2016.

[4] Y. Koyamada, M. Imahama, K. Kubota, and K. Hogari, "Fiber-optic distributed strain and temperature sensing with very high measurand resolution over long range using coherent OTDR," J. Lightw. Technol., vol. 27, no. 9, pp. 1142-1146, Apr. 2009.

[5] T. Shiota and F. Wada, "Distributed temperature sensors for single mode fibers," Proc. SPIE, vol. 1586, pp. 13-18, 1991.
[6] L. Thévenaz, A. Fellay, M. Facchini, W. Scandale, M. Nikles, and P. A. Robert, "Brillouin optical fiber sensor for cryogenic thermometry," Proc. SPIE, vol. 4694, pp. 22-27, 2002.

[7] C. Lupi, F. Felli, A. Brotzu, M. A. Caponero, and A. Paolozzi, "Improving FBG sensor sensitivity at cryogenic temperature by metal coating," IEEE Sensors J., vol. 8, no. 7, pp. 1299-1304, Jul. 2008

[8] X. Lu, M. A. Soto, and L. Thévenaz, "Temperature sensitivity enhancement in a standard optical fiber with double coatings at low temperature," Proc. SPIE, vol. 10323, p. 1032371, 2017.

[9] T. Habisreuther et al., "ORMOCER coated fiber-optic Bragg grating sensors at cryogenic temperatures," IEEE Sensors J., vol. 12, no. 1, pp. 13-16, Jan. 2012.

[10] H. Gu, H. Dong, G. Zhang, J. He, and H. Pan, "Effects of polymer coatings on temperature sensitivity of Brillouin frequency shift within doublecoated fibers," IEEE Sensors J., vol. 13, no. 2, pp. 864-869, Feb. 2013.

[11] C. D. Boyd, B. D. Dickerson, and B. K. Fitzpatrick, "Monitoring distributed temperatures along superconducting degaussing cables via Rayleigh backscattering in optical fibers," in Proc. Intell. Ships Symposium IX, 2011.

[12] F. Scurti, J. McGarrahan, and J. Schwartz, "Effects of metallic coatings on the thermal sensitivity of optical fiber sensors at cryogenic temperatures," Opt. Mater. Exp., vol. 7, no. 6, pp. 1754-1766, Jun. 2017.

[13] S. R. Schmid and A. F. Toussaint, "Optical fiber coatings," in Specialty Optical Fibers Handbook. Burlington, NJ, USA: Academic, 2007. pp. 95-122.

[14] N. Lagakos, J. A. Bucaro, and J. Jarzynski, "Temperature-induced optical phase shifts in fibers," Appl. Opt., vol. 20, no. 13, pp. 2305-2308, Jul. 1981.

[15] Y. Nakajima et al., "A study for estimating thermal strain and thermal stress in optical fiber coatings," Furukawa Rev., vol. 34, pp. 8-12, 2008.

[16] M. Okaji, N. Yamada, K. Nara, and H. Kato, "Laser interferometric dilatometer at low temperatures: Application to fused silica SRM 739," Cryogenics, vol. 35, no. 12, pp. 887-891, Dec. 1995.

[17] Y. Yang, H Lee, and H. Chou, "Elasto-optics in double-coated optical fibers induced by axial strain and hydrostatic pressure," Appl. Opt., vol. 41, no. 10, pp. 1989-1994, Apr. 2002.

[18] J. H. Lee, "High nonlinearity optical fiber technology for all-optical signal processing devices," in Progress in Optical Fibers Research. New York, NY, USA: Nova, 2007, pp. 105-177.

[19] S. S. Reddy, B. J. Overton, and S. M. Watson, "Design parameters of coatings for low-temperature applications of optical fibers," presented at the Opt. Fiber. Commun. Conf., San Diego, CA, USA, 1995, Paper ThH5.

[20] S. Le Floch and P. Cambon, "Study of Brillouin gain spectrum in standard single-mode optical fiber at low temperatures $(1.4-370 \mathrm{~K})$ and high hydrostatic pressures (1-250 bars)," Opt. Commun., vol. 219, no. 1, pp. 395-410, Jan. 2003.

[21] H. Gu, H. Dong, G. Zhang, Y. Dong, and J. He, "Dependence of Brillouin frequency shift on radial and axial strain in silica optical fibers," Appl. Opt., vol. 51, no. 32, pp. 7864-7868, Nov. 2012.

[22] S. B. Mahar, "Spontaneous Brillouin scattering quench diagnostics for large superconducting magnets," Ph.D. dissertation, Dept. Nucl. Sci. Eng., Massachusetts Inst. Technol., Cambridge, MA, USA, 2008.

[23] M. Tur et al., "Structural health monitoring of composite-based UAVs using simultaneous fiber optic interrogation by static Rayleigh-based distributed sensing and dynamic fiber Bragg grating point sensors," Proc. SPIE, vol. 9634, p. 96340P, 2015.

[24] M. Froggatt and J. Moore, "High-spatial-resolution distributed strain measurement in optical fiber with Rayleigh scatter," Appl. Opt., vol. 37, no. 10, pp. 1735-1740, Apr. 1998

[25] X. Lu, M. A. Soto, and L. Thévenaz, "Temperature-strain discrimination in distributed optical fiber sensing using phase-sensitive optical time-domain reflectometry," Opt. Exp., vol. 25, no. 14, pp. 16059-16071, Jun. 2017.

[26] A. D. Kersey et al., "Fiber grating sensors," J. Lightw. Technol., vol. 15, no. 8, pp. 1442-1463, Aug. 1997.

[27] D. B. Leviton and B. J. Frey, "Temperature-dependent absolute refractive index measurements of synthetic fused silica," Proc. SPIE, vol. 6273, p. $62732 \mathrm{~K}, 2006$.

[28] S. W. James, R. P. Tatam, A. Twin, M. Morgan, and P. Noonan, "Strain response of fibre Bragg grating sensors at cryogenic temperatures," Meas. Sci. Technol., vol. 13, pp. 1535-1539, Sep. 2002.

[29] X. Lu, M. A. Soto, and L. Thévenaz, "MilliKelvin resolution in cryogenic temperature distributed fibre sensing based on coherent Rayleigh scattering," Proc. SPIE, vol. 9157, p. 91573R, 2014

[30] M. A. Soto and L. Thévenaz, "Modeling and evaluating the performance of Brillouin distributed optical fiber sensors," Opt. Exp., vol. 21, no. 25, pp. 31347-31366, Dec. 2013. 
[31] T. Fildhuth, "Design and monitoring of cold bent lamination-stabilised glass investigated by applying fibre optic sensors," Ph.D. dissertation, Inst. Build. Struct. Structural Design, Universitat Stuttgart, Stuttgart, Germany, 2015.

[32] J. M. Gere, Mechanics of Materials, 6st ed. Belmont, W.A., Australia: Thomson-Engineering, 2003, Appendix H, pp. 911-915.

[33] M. A. Soto, S. Chin, and L. Thévenaz, "Double-pulse Brillouin distributed optical fiber sensors: Analytical model and experimental validation," Proc. SPIE, vol. 8421, p. 842124, 2012.

Xin $\mathbf{L u}$ received the M.Sc. degree in optical engineering from the University of Electronic Science and Technology of China, Chengdu, China, in 2011, and the Ph.D. degree in electrical engineering from the Swiss Federal Institute of Technology of Lausanne, Lausanne, Switzerland, in 2016. Since November 2016, he has been with the Institute for Astronomy, Swiss Federal Institute of Technology, Zurich, Switzerland, as a Postdoctoral Researcher. His main research interests include distributed fiber sensing, nonlinearities in optical fibers, adaptive optics, and high-contrast imaging.

Marcelo A. Soto received the M.Sc. degree in electronic engineering from Universidad Técnica Federico Santa María (UTFSM), Valparaíso, Chile, in 2005, and the Ph.D. degree in telecommunications from Scuola Superiore Sant'Anna (SSSA), Pisa, Italy, in 2011.

In 2006, he was a Lecturer in the Department of Electronic Engineering, UTFSM. During 2010-2011, he was a Research Fellow with SSSA, where he worked on distributed optical fiber sensors based on Raman and Brillouin scattering. From November 2011, he is a Postdoctoral Researcher with the Swiss Federal Institute of Technology of Lausanne, Lausanne, Switzerland, where he has been working on high-performance Brillouin and Rayleigh distributed fiber sensing, nonlinear fiber optics, optical signal processing, and optical Nyquist pulse generation. He is author or coauthor of about 140 scientific publications in international refereed journals and conferences in the fields of optical communications and optical fiber sensing.

Dr. Soto is a Member of the Optical Society of America, and he is in the Board of Reviewers of major international journals in photonics.
Luc Thévenaz (M'01-SM'12-F'17) received the M.Sc. and Ph.D. degrees in physics from the University of Geneva, Geneva, Switzerland.

In 1988, he joined the Swiss Federal Institute of Technology of Lausanne, Lausanne, Switzerland, where he currently leads a research group involved in photonics, namely fiber optics and optical sensing. He achieved with his collaborators the first experimental demonstration of optically controlled slow and fast lights in optical fibers, realized at ambient temperature and operating at any wavelength since based on stimulated Brillouin scattering. He also contributed to the development of Brillouin distributed fiber sensing by proposing innovative concepts pushing beyond barriers. During his career, he was with Stanford University, with Korea Advanced Institute of Science and Technology, with Tel Aviv University, with the University of Sydney, and with the Polytechnic University of Valencia. In 2000, he cofounded the company Omnisens that is developing and commercializing advanced photonic instrumentation based on distributed fiber sensing. He is an Associate Editor of three major scientific journals. His research interests include Brillouin-scattering fiber sensors, slow and fast lights, nonlinear fiber optics, and laser spectroscopy in gases.

Dr. Thévenaz is a Fellow of the Optical Society of America. 